\title{
Guillain Barré Syndrome in a three year old child under treatment for atypical febrile seizure: A case report
}

\section{Basnet R', Subedi $K^{2}$, Bhandari $\mathrm{B}^{3}$, Gongal $\mathrm{S}^{4}$}

${ }^{1}$ Rydam Basnet, Lecturer; ${ }^{2}$ Kiran Subedi, Resident; ${ }^{3}$ Bikash Bhandari, Resident; ${ }^{4}$ Sonsy Gongal, Resident; Department of Paediatrics, Kathmandu Medical College Teaching Hospital, Kathmandu, Nepal

\begin{abstract}
Guillain-Barré syndrome is the most common cause of acute flaccid paralysis in healthy infants and children. It has an annual incidence of 0.6 to 2.4 cases per 100,000 population and occurs at all ages and in both sexes. The incidence is lower in children ranging from 0.38 to 0.91 cases per 100,000 in the studies and most of these cases ranging from four years to 18 years of age. Hence, the true incidence is even lesser in child younger than four years.

We report a case of an early presentation of Guillain-Barré syndrome in child who was also a case of atypical febrile seizure under sodium valproate. Keeping in mind all the possibilities of acute onset weakness including orthopedic problems, possible side effect of valproate and possible new seizure, treatment was started early solely based on history and physical examination later confirmed by laboratory investigations.
\end{abstract}

Key words: acute flaccid paralysis, albuminocytologic dissociation, Guillain-Barré syndrome, intravenous immunoglobulin

\section{INTRODUCTION}

ince polio is being successfully eliminated from most $\checkmark$ part of the world, Guillain-Barré syndrome(GBS), a rare disease has now become the most common cause of acute flaccid paralysis (AFP) in the world ${ }^{1}$. This disease is known to cause life threatening complications. Laboratory investigation are only helpful in later part of the disease. It is extremely important to identify the disease early which can be done on clinical grounds. Intravenous immunoglobulin (Ivlg) is available for its treatment but is an expensive drug. This drug should be started early to avoid life threatening complications. So this case highlights the importance of clinical examination based early initiation of treatment to successfully manage a case of GBS.

\section{CASE REPORT}

According to the father, the child was doing well in the morning, playing around, but in the evening, the father noticed the child was unable to walk and fell down

Address for correspondence

Dr. Rydam Basnet

Lecturer, Department of Paediatrics

Kathmandu Medical College Teaching Hospital

Sinamangal,Kathmandu, Nepal

E-mail: rydam@hotmail.com while trying to do so, but the child was able to move his bilateral (b/l) limbs while at rest. After that the child was rested by the parents but the symptom persisted till next morning and child was brought immediately to the hospital. There was history of (h/o) preceding diarrhoea three weeks back, 6-7 episodes a day, watery in consistency, not associated with fever or dehydration, no blood mixed and lasted for around two weeks which was managed with oral rehydration solution (ORS) as stool reports were normal. There was no h/o trauma, excessive crying, fever, rash, seizure, vomiting, cough and was passing urine and stool normally, neither was there a h/o dog bite, difficulty in swallowing, recent vaccination nor headache, blurred vision, tinnitus, vertigo, dysphagia, or incontinence.

The child was previously diagnosed as a case of atypical febrile convulsion at seven months of age and was under sodium valproate. Later the dose was tapered to $12 \mathrm{mg} /$ kg. Electroencephalography (EEG) records four months back was suggestive of seizure disorder. The child was seizure free since 25 months of age.

The child was born via caeserean section at hospital at term, birth weight of $3 \mathrm{~kg}$ with no postnatal complications and immunised as per national immunisation programme. The child had attained 
normal developmental milestones as per age.

On examination vitals were stable, the child was well nourished, conscious, oriented to person, place and sitting in mother's lap.

\section{CNS EXAMINATION}

- Cranial nerves: intact

\section{- Motor examination:}

bulk: bulk on b/l upper and lower limbs within normal limit

tone: tone on b/l upper limbs within normal limit but decreased tone on $b / l$ lower limbs

power: power on b/l upper limbs were $4 / 5$ and that on b/l lower limbs were $2 / 5$

reflexes: normal on b/l upper limbs but ankle and knee reflexes were absent on both the lower limbs.

Sensations were intact. Bilateral planter reflexes were mute. Gait could not be assessed. There were no abnormalities detected in cardiac, respiratory and gastrointestinal system.

Preliminary investigations such as complete blood count, thyroid function test and serum electrolytes were normal. Valproic acid level was within therapeutic range. Stool sample was sent for AFP Surveillance which was later normal.

During the admission period mother complained of episodes of increased sweating. Muscle weakness gradually progressed involving the trunk and the upper limbs. The child was not able to sit without support and was later unable to raise his hands. Based on history, physical findings and disease progression a diagnosis of GBS was made.

Since the ascending paralysis can rapidly involve respiratory muscles, a decision to start intravenous immunoglobulin was made which was based solely on the clinical symptoms, as albuminocytologic dissociation takes time to develop. Ivlg $0.4 \mathrm{~g} / \mathrm{kg} /$ day for 5 consecutive days was given.

Lumbar puncture was performed two days later which showed no cells, sugar: $46 \mathrm{mg} / \mathrm{dl}$ and protein: $1864 \mathrm{mg} / \mathrm{l}$ showing elevated cerebrospinal fluid (CSF) protein with a normal CSF white blood count (WBC) count. This finding known as albuminocytologic dissociation, presents in about 50 to 66 percent of patients with GBS in the first week after the onset of symptoms and $\geq 75$ percent of patients in the third week.

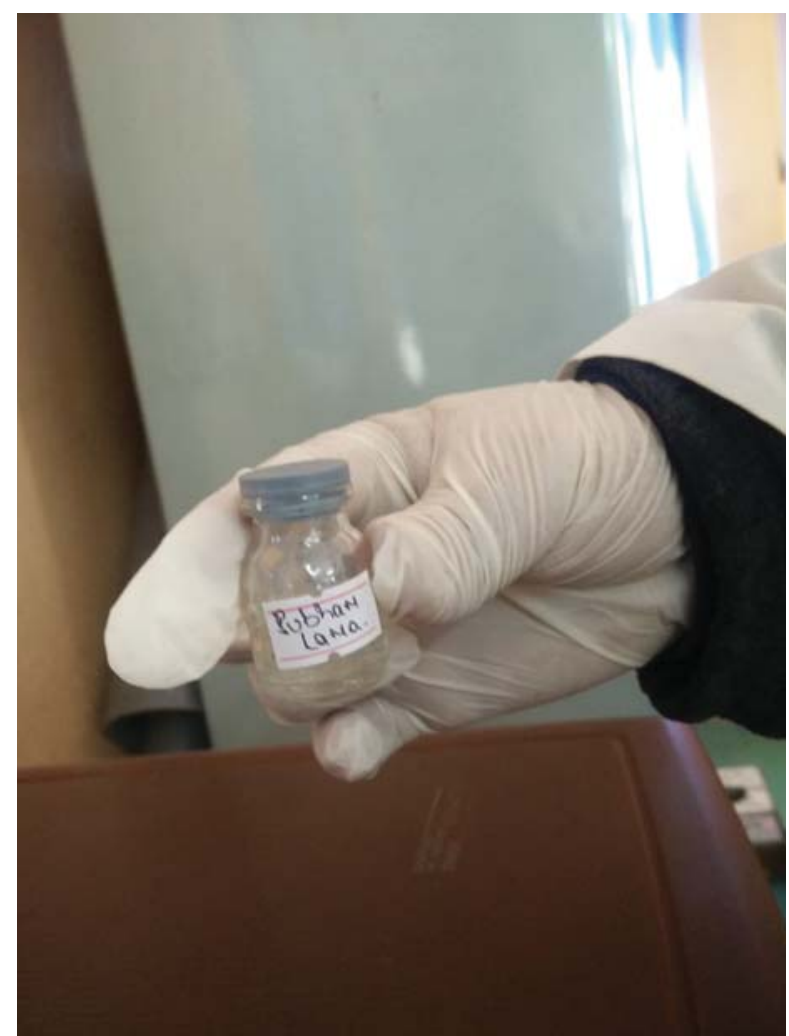

Figure 1: Cerebrospinal fluid (CSF) sample of the patient (Transparency: clear)

After the fourth dose of Ivlg the patient showed some signs of improvement. The child was able to raise his hands above the shoulder and was later able to sit and stand with support. Based on the improving symptoms the child was discharged. The child came on regular follow up, was able to walk without support after two weeks of discharge and later began to carry out his normal activities.

\section{DISCUSSION}

In patients with acute inflammatory demyelinating polyradiculopathy (AIDP), the most common form of GBS, two-thirds develop the neurologic symptoms two to four weeks after having what appears to be a benign febrile respiratory or gastrointestinal infection as in our case ${ }^{2}$. The concurrent use of sodium valproate and atypical febrile seizure in the past created diagnostic dilemma to us. An Indian case-control study reported that $27.7 \%$ of childhood GBS cases were associated with $C$ jejuni infection ${ }^{3}$. A study in Iran showed that $47 \%$ of paediatric GBS cases had evidence of recent $C$ jejuni infection. Since the disappearance of polio in 2000 in Bangladesh, a high incidence of acute flaccid weakness in Bangladeshi children $(3.25$ cases per 100,000) is still present but is now related mostly to GBS. Frequent exposure to enteric 
pathogens at an early age may increase this incidence of $\mathrm{GBS}^{5}$. Recent retrospective reviews of childhood GBS reported the average age to be in the range of 4-8 years ${ }^{6}$. Delay in diagnosis in preschool children $(<6$ years) may occur because preschool children usually appear with refusal to walk and pain in the legs, whereas older children (aged 6-18 years) present with more classic symptoms (weakness and paraesthesia). This often leads to initial misdiagnosis in preschool children with myopathy, tonsillitis, meningitis, rheumatoid disorders, coxitis, or diskitis ${ }^{6}$. In general, the outcome of GBS is more favourable in children than in adults, however, the recovery period is long, often weeks to months. Rarely, it can be fatal in $5-10 \%$ of patients with respiratory failure and cardiac arrhythmia ${ }^{7}$. In patients with GBS, lumbar puncture often reveals an elevated CSF protein with a normal CSF white blood cell count. This finding, known

\section{REFERENCES}

1. Van Doorn PA, Ruts $L$, Jacobs BC.Clinical features, pathogenesis, and treatment of Guillain-Barré syndrome.Lancet Neurol. 2008;7:939-50.

2. Hahn AF. Guillain-Barré syndrome. Lancet. 1998;352:635.

3. Kalra V, Chaudhry R, Dua T, Dhawan B, Sahu JK, Mridula B. Association of Campylobacter jejuni infection with childhood Guillain-Barré syndrome: a case-control study. J Child Neurol. 2009 Jun; 24(6):664-8.

4. Barzegar M, Alizadeh A, Toopchizadeh V, Dastgiri S, Majidi J. Association of Campylobacter jejuni infection and GuillainBarré syndrome: a cohort study in the northwest of Iran. Turk J Pediatr. 2008 Sep-Oct;50(5):443-8. as albuminocytologic dissociation, is present in 50 to 66 percent of patients with GBS in the first week after the onset of symptoms and $\geq 75$ percent of patients in the third week ${ }^{8}$.

\section{CONCLUSION}

Hence acute onset paralysis in a child requires meticulous history, clinical examination and investigation to rule out potentially life threatening conditions such as Guillian-Barré syndrome. GuillianBarré syndrome can be diagnosed by history and physical examination alone and treatment at times has to be started early to avoid life threatening complications without awating CSF findings which takes longer to develop. In our case, Ivlg was instituted early in the course of illness which led to the resolution of symptoms within a few days.

5. Islam Z, Jacobs BC, Islam MB, Mohammad QD, Diorditsa S, Endtz HP. High incidence of GuillainBarre syndrome in children, Bangladesh. Emerg Infect Dis. 2011 Jul;17(7):1317-8.

6. Roodbol J, de Wit MC, Walgaard C, de Hoog M, Catsman-Berrevoets $C E$, Jacobs BC. Recognizing Guillain-Barre syndrome in preschool children. Neurology. 2011 Mar 1. 76(9):807-10.

7. Lee JH, Sung IY, Rew IS. Clinical presentation and prognosis of childhood Guillain-Barré syndrome.J Paediatr Child Health. 2008 Jul-Aug;44(7-8):449-54.

8. Nishimoto Y, Odaka M, Hirata K, Yuki N. Usefulness of anti-GQ1b IgG antibody testing in Fisher syndrome compared with cerebrospinal fluid examination. $J$ Neuroimmunol. 2004;148:200. 